\title{
Zeitmanagement der operativen Therapie - was wird wann operiert?
}

Maria Alexandra Bernstorff, Uwe Hamsen, Christian Waydhas

\section{Einleitung}

Ein polytraumatisierter Patient bedarf einer komplexen und gut strukturierten Behandlung. Diese muss vom Ersthelfer über den Rettungsdienst, ggf. über das erstversorgende Krankenhaus bis hin zur endgültigen Behandlung im Zielkrankenhaus geplant werden. In der Klinik wird der Patient über den Schockraum aufgenommen und die Verletzungen der Schwere nach triagiert und behandelt. Bei Häusern, die nicht alle Fachabteilungen zur endgültigen Versorgung des Patienten besitzen, ist an eine sekundäre Verlegung zu denken.

Eine Schockraumversorgung sollte in jedem Haus nach einem standardisierten Schema ablaufen, um vorzubeugen, Verletzungen zu übersehen. Hier bietet sich das ABCDE-Schema nach ATLS (Advanced Trauma Life Support) an. Es bietet einen strukturierten Ablauf für die Behandlung eines Patienten in der Notfallsituation. Dabei wird mit dem potenziell lebensbedrohlichsten Bereich begonnen, wobei A für Airway, B für Breathing, C für Circulation, D für Disability und E für Environement bzw. Exposure stehen.

In dieser ersten Phase der Polytraumaversorgung stellen sich vor allem die Fragen: „Welche Verletzung bedarf als Erstes der Versorgung?“ „Benötigt der Patient eine operative Versorgung?“ „Ist der Patient stabil genug für eine Operation?" (Deutsche Gesellschaft für Unfallchirurgie, S3-Leitlinie/Schwerverletztenbehandlung [1]). Die Triagierung des Patienten folgt dem Prinzip: „Treat first, what kills first“, ggf. sogar unter sofortiger Unterbrechung der Diagnostik bei akuter vitaler Bedrohung des Patienten.

\section{Primärversorgung}

Bei der Primärversorgung werden die potenziell vital bedrohlichen Zustände zuerst behoben. Die Aspekte der unter den Kategorien „A“ und des „B“ im ABCDE-Schema subsumierten Einschränkungen werden zumeist bereits im Schockraum bzw. der Notaufnahme behandelt. Hierbei kann es notwendig sein, einen Patienten zu intubieren oder ihm eine Thoraxdrainage zur schnellen Entlastung eines Pneumo- oder Hämatothoraxes zu legen. Chirurgischer Interventionsbedarf im Sinne einer operativen Versorgung beginnt zumeist bei der Behandlung der unter „C“ zusammengefassten Verletzungen, wobei es sich häufig um Blutungen mit Einschränkung der Kreislaufsituation handelt. Hämodynamisch relevante Blutungen müssen sofort versorgt werden, um dem Patienten ein Überleben zu sichern. In seltenen Fällen mit schwerstem Blutungsschock oder bei einer Reanimationssituation nach Trauma kann eine Notfallthorakotomie oder Notfalllaparotomie ggf. noch im Schockraum erforderlich sein. Im Anschluss sollten notwendige Operationen bei schweren Kopf - und offenen Hohlorganverletzungen sowie offene Frakturen operiert werden. Die in diesem Zusammenhang häufig auffallenden Haut- und Weichteilverletzungen sollten ebenso nach Dringlichkeit triagiert und versorgt werden. Bei Quetschtraumen häufig auftretende Kompartmentsyndrome gehören ebenfalls zu den unmittelbar zu versorgenden Notfällen. Grundsätzlich unterscheidet man zwischen der frühen primären Versorgung, dem „early-total-care“-Prinzip und dem Konzept des „damage control“, bei dem nur unbedingt notwendige chirurgische Eingriffe zur Stabilisierung der Vitalparameter durchgeführt werden [2].

Das „early-total-care“-Konzept wurde vermehrt in den späten $80 \mathrm{er}$ - und frühen $90 \mathrm{er}-J a h r e n$ angewendet. Es beinhaltet die definitive chirurgische Versorgung aller Frakturen langer Röhrenknochen und von Beckenbrüchen innerhalb der ersten 24-48 h [3]. Grundgedanke dieser Idee war die Reduktion der Gefahr von Fettembolien, die von Frakturen langer Röhrenknochen ausgehen können. Hierdurch lässt sich das Risiko respiratorischer Komplikationen minimieren. Die Möglichkeit einer frühen Mobilisation reduziert zusätzlich die Aufenthaltsdauer auf Intensivstation, was die Komplikationsrate und das Auftreten von Begleiterkrankungen senkt [4]. Eingeräumt werden muss jedoch, dass das Operationsrisiko deutlich erhöht ist. Man fügt dem Patienten in einer sehr vulnerablen Phase ein erneutes Trauma zu. Aus pathophysiologischer Sicht entspricht das Operationstrauma qualitativ dem Unfalltrauma (Näheres zur Pathophysiologie s.u.).

Anders ist dies beim Prinzip des „damage control“. Ursprünglich ist dies ein Begriff aus der Abdominalchirurgie, bei dem ein schwer traumatisierter Patient abdominell so stabilisiert wird, dass eine aktive Blutung oder eine Hohlorganperforation durch den möglichst geringsten operativen Eingriff bestmöglich kontrolliert wird. So können Blutungen tamponiert werden („Packing“, Einlegen von Bauchtüchern zur Tamponade aktiver Blutungen) [5]. 
Damit kann die Operationszeit möglichst kurz gehalten und der Patienten so schnell wie möglich auf die Intensivstation zur weiteren Stabilisierung verlegt werden. Das gleiche Prinzip wurde in die Unfallchirurgie zur Versorgung langer Röhrenknochen und Beckenfrakturen übertragen. Das Konzept des „damage control orthopedics“ beinhaltet 4 Phasen der Traumaversorgung. Nach der 1. Phase der Stabilisierung bei akut lebensbedrohlichen Verletzungen erfolgt direkt in der 2. Phase die temporäre operative Stabilisierung langer Röhrenknochen sowie eventueller Beckenfrakturen. Dies geschieht zumeist mit einem Fixateur externe. Zusätzlich werden Weichteilverletzungen entsprechend gereinigt und versorgt. Hierzu gehört ein Débridement sämtlicher offenen Wunden sowie ein entweder temporärer Wundverschluss mit z.B. Wundauflagen oder eine Vakuumtherapie oder bereits ein direktes Verschließen der Wunden. Den Beginn der 4. Phase zu definieren, bedarf einer guten klinischen Beurteilung des Patienten. Diese definiert den Zeitpunkt sekundärer Operationen und der damit einhergehenden definitiven chirurgischen Versorgung von zuvor nur temporär versorgten Verletzungen [6].

Das zeitliche Management sekundärer Operationen ist ein komplexer Prozess, der trotz definierter Standards einer hohen Individualisierung bedarf [7].

\section{Dringliche Operationen}

In der Regel gilt, dass Verletzungen, die sich im Verlauf hämodynamisch relevant zeigen oder aber ein schlechtes neurologisches Outcome des Patienten bei Schädel-HirnTraumen bei Nichtversorgung erwarten lassen, nicht in Abhängigkeit von o.g. Kriterien operativ versorgt werden.

Akute intrakranielle Blutungen sind gekennzeichnet durch eine Primär- und Sekundärverletzung. Die Primärverletzung erfolgt durch die initiale direkte Krafteinwirkung auf das Hirnparenchym [8]. Bei Primärverletzungen erfolgt die operative Versorgung zumeist direkt nach der Diagnostik, entweder in Form einer operativen Hämatomausräumung, einer dekompressiven Kraniektomie oder durch Einsetzen einer intrakraniellen Sonde zur intrakraniellen Hirndruckmessung (ICP-Messung) für den Verlauf der intensivmedizinischen Behandlung.

Die ICP-Messung dient vor allem dem Monitoring von Sekundärverletzungen, die aus einer Kombination von Gefäß- und Nervenschädigungen, Ischämie, Inflammation und freien Radikalen entstehen [8]. Zusätzlich zur ICPMessung erfolgt eine engmaschige klinische Überwachung der Pupillenreaktion und Kontrolle durch Prüfung des Glasgow-Coma-Scales (sofern der Patient nicht in Narkose ist) und der Stammhirnreflexe. Der Zeitpunkt einer operativen Versorgung muss im Falle des SchädelHirn-traumatisierten Patienten direkt bei klinischer Auf- fälligkeit erfolgen, unabhängig von allgemein gültigen Kriterien der Operabilität.

Ähnlich verhält es sich mit abdominellen oder thorakalen Verletzungen. Bei größeren abdominellen Verletzungen erfolgt eine direkte operative Versorgung. Sollte in der initialen Sonografie oder Computertomografie kein Anhalt für eine abdominelle Verletzung bestehen, ist eine engmaschige Kontrolle dennoch wichtig. Während der ersten Stunden und Tage sollten regelmäßig klinische sowie laborchemische Untersuchungen erfolgen. Beim Tertiary Survey kann neben der klinischen Untersuchung eine abdominelle und thorakale Sonografie durchgeführt werden in Hinblick auf mögliche verzögert auftretende freie Flüssigkeit, auch ohne Anhalt einer klinischen Verschlechterung.

Warnsignale sind abdomineller Druckschmerz, Abwehrspannung, starker Anstieg der Leukozyten, Transaminasen oder der Pankreasenzyme, neu aufgetretene freie Flüssigkeit in der Sonografie sowie starker gastraler Reflux. Zur weiteren Abklärung können eine erneute Computertomografie, eine Probepunktion freier Flüssigkeit, die Laparoskopie und in Einzelfällen eine Probelaparotomie infrage kommen. Bei Verdacht auf Pankreasverletzungen ist eine ERCP (endoskopisch retrograde Cholangiopankreatikografie) in einigen Fällen sinnvoll. Beim Verdacht auf oder bei Feststellen von freier Flüssigkeit ist das Kreislaufmonitoring besonders wichtig. Tritt durch intraabdominellen Blutverlust eine Kreislaufinstabilität des Patienten ein, ist dies eine absolute und sofortige Indikation zur Intervention, sei es operativ oder, wenn die Möglichkeit besteht, angiografisch-interventionell.

Ebenfalls eine sofortige OP-Indikation stellt das abdominelle Kompartmentsyndrom dar. Dies ist definiert durch einen persistierend erhöhten intraabdominellen Druck $>20 \mathrm{mmHg}$ in Kombination mit neu aufgetretenen Organfunktionsstörungen (Verschlechterung der Lungenund oder Nierenfunktion, Hypotension, Anstieg des Hirndrucks).

Bei thorakalen Verletzungen besteht zumeist eine direkte Operationsindikation, wenn es sich bspw. um Abrisse oder Einrisse größerer Gefäße oder eine Herzbeuteltamponade handelt. Ein Hämato- oder Pneumothorax kann zumeist direkt auf der Intensivstation mit einer Thoraxdrainage versorgt werden, wenn dies nicht bereits präklinisch oder im Schockraum erfolgt ist.

Die rechtzeitige Erkennung eines Kompartmentsydroms in Muskellogen der Extremitäten und das richtige zeitliche Management bedürfen einer guten klinischen Einschätzung und einer hohen Aufmerksamkeit. Prinzipiell gilt, dass ein Muskellogensyndrom entlastet werden muss, bevor es zu irreversiblen Gewebenekrosen kommt. Das Erkennen eines Kompartmentsyndroms ist insbeson- 
dere beim wachen und ansprechbaren Patienten vor allem eine klinische Diagnose. Während der starke Schmerz noch als frühes Symptom gewertet werden kann, weisen das Auftreten von Pulslosigkeit, Blässe, Parästhesien und Lähmungen auf bereits eingetretene schwere Gewebeschädigungen oder Nekrosen hin. Im Verdachtsfall kann die Messung der Drücke in den einzelnen Muskellogen die Diagnose sichern. Bei bewusstlosen oder sedierten Patienten, wie häufig nach Polytrauma, ist die Druckmessung neben dem klinischen Befund einer verhärteten Muskelloge für die Diagnosestellung oft entscheidend. Besonders schwierig ist die Entscheidung oft, wenn die Kompartmentdrücke in einem Graubereich zwischen normal und eindeutig zu hoch liegen und die entsprechende Klinik nicht eindeutig oder aufgrund eines Komazustandes nicht wegweisend ist. Irreversible Myonekrosen sind bereits 3-4 h nach Trauma nachweisbar, nahezu immer nach $8 \mathrm{~h}$. Deswegen erscheint eine frühzeitige Dermatofasziotomie trotz der dadurch bedingten Morbidität und der Notwendigkeit von plastisch-rekonstruktiven Folgeeingriffen eher vorteilhaft. Die aktuelle Literatur bestätigt, dass in der Nutzen-Schaden-Abwägung die frühzeitige Dermatofasziotomie günstiger ist als ein übersehenes oder zu spät entlastetes Kompartmentsyndrom. Besonderes Augenmerk benötigen auch die sekundären Kompartmentsyndrome nach operativer Versorgung oder im Zuge einer größeren Fraktur, ebenso wie die leicht zu übersehenden Kompartmentsyndrome am Fuß [9].

\section{Sekundäroperationen}

Aus pathophysiologischer Sicht entspricht das Operationstrauma qualitativ weitgehend dem Unfalltrauma. Dies ist klinisch gekennzeichnet durch einen Hypermetabolismus und eine systemische Entzündungsreaktion (Fieber, Anstieg der Herzfrequenz und des Sauerstoffverbrauches, Leukozytose etc.). Es konnten zahlreiche Reaktionsmuster des endokrinen Systems mit biochemischen Folgereaktionen festgestellt werden. Beispiele hierfür sind ein Anstieg des Adrenalin- und Kortisolspiegels sowie zahlreicher anderer Hormone, eine Negativierung der Stickstoffbilanz, eine Aktivierung der Granulozyten sowie ein Anstieg proinflammatorischer (Interleukin 2, 6 und 8) sowie antiinflammatorischer Zytokine (Interleukin 10). All diese Reaktionen stehen in Abhängigkeit zur Größe und Schwere des Unfalltraumas. Dies konnte in gleicher Weise auch für das Operationstrauma nachgewiesen werden. Zudem konnte gezeigt werden, dass postoperative Entzündungsreaktionen nach Femurnagelung, Totalendoprothesenimplantationen an der Hüfte, Beckenosteosynthesen und vergleichbare Eingriffe ähnlich derer sind, die nach mittelschweren Mehrfachverletzungen (Injury Severity Score [ISS] bis 24 Punkte) ausgelöst werden.

Um den richtigen Zeitpunkt der Versorgung eines schwer traumatisierten Patienten abschätzen zu können, ist es wichtig, die Kompromittierung der Homöstase, Organfunktionen und Kreislaufsituation in den verschiedenen Phasen nach einem Trauma zu verstehen. Zu allererst geht es in der Intensivmedizin um ein gutes Zusammenspiel zwischen kausaler Therapie der Grunderkrankung und Unterstützung von gestörten Organfunktionen und der symptomatischen Therapie der Homöstase. Behandlungsansätze für die kausale Therapie eines polytraumatisierten Patienten beinhalten die Blutungsstillung, die Beseitigung von Kompartmentsyndromen, die Organerhaltung, wiederherstellende und rekonstruktive Eingriffe und die Fokussanierung. Symptomatische Therapien unterstützen diese Behandlung, bis die kausale Therapie zusammen mit der Selbstheilung zur Wiederherstellung der Organ- und Kreislauffunktion ohne Unterstützung greift. Während am Anfang Schock- und Schockfolgereaktionen sowie Blutungen und Gerinnungsstörungen im Vordergrund stehen, entwickeln sich in der Folgezeit Infekte bis hin zur Sepsis.

Die nachfolgende $>$ Tab. 1 gibt einen Überblick über Kriterien, die Indikatoren für einen Übertritt des Patienten aus der inflammatorisch-vulnerablen Phase in ein Stadium der Stabilisierung mit dem Überwiegen reparativer und regenerativer Vorgänge sind. Wichtig ist zu betonen, dass man einen Patienten mit seinen Labor- und Vitalwerten nicht absolut betrachten sollte. Sieht man sich die Werte im Verlauf an, lässt sich häufig ein Plateau erkennen und man kann in der Relation der Voruntersuchungen feststellen, ab wann der Zustand sich stabilisiert oder sogar bessert.

Im Allgemeinen zeigen die o.g. Richtwerte eine Operabilität des Patienten an. Es wird jedoch immer mehr dazu übergegangen, eine möglichst frühe Versorgung anzustreben. Labor- oder Vitalwerte sollten deshalb nicht als Absolutwerte gesehen werden. Wichtig ist, den Verlauf genau zu beobachten: Nimmt die Leukozytenzahl bereits wieder ab? Stabilisieren sich die Thrombozyten? Nimmt die Compliance der Lunge bereits wieder zu? Beobachtet man klinisch sowie anhand von Labor- und Vitalwerten, dass ein Peak erreicht und bereits wieder eine Regeneration zu beobachten ist, sollte frühzeitig an eine definitive Versorgung von Frakturen gedacht werden. Besonders schwer verletzte Patienten haben ein hohes Risiko, Komplikationen wie Pneumonie, Lungenembolie oder eine Sepsis zu erleiden. Daher sind eine frühe Mobilisation und eine Verkürzung der Intensivzeit anzustreben.

Idealerweise werden die Frakturen so versorgt, dass eine Mobilisierung des Patienten frühzeitig möglich ist. Hierfür sollten die Frakturen dreh- und lagerungsstabil versorgt werden. Von einer Ruhigstellung im Gips oder mit Orthesen ist aufgrund der schnellen Neigung zu Ödemen und Hämatomen abzuraten. 
- Tab. 1 Kriterien der Operabilität beim polytraumatisierten Intensivpatienten [10, 14].

\begin{tabular}{|c|c|}
\hline allgemein & $\begin{array}{l}\text { - keine sekundären Operationen an Tag } 2 \text { und } 3 \text { nach Trauma } \\
\text { - keine akuten Komplikationen (außer sie bedürfen einer Operation) } \\
\text { - alle Organfunktionen stabil mit Tendenz zur Verbesserung } \\
\text { - Reduzierung der organunterstützenden Therapiemaßnahmen }\end{array}$ \\
\hline Inflammation/Infektion & $\begin{array}{l}\text { - C-reaktives Protein fallend } \\
\text { - Thrombozytenzahl }>100 / \mathrm{nl} \text { und steigend } \\
\text { - Temperatur }<38,5^{\circ} \mathrm{C}(\text { Tendenz gleich oder fallend) } \\
\text { - keine klinischen, laborchemischen oder radiologischen Zeichen einer floriden Sepsis }\end{array}$ \\
\hline Lungenfunktion & $\mathrm{PaO}_{2} / \mathrm{FiO}_{2}>280$ (Tendenz gleichbleibend oder steigend), PEEP $<15 \mathrm{mbar}$ \\
\hline Kreislauf- und Nierenfunktion & $\begin{array}{l}\text { - negative oder ausgeglichene Flüssigkeitsbilanz innerhalb der letzten } 48 \mathrm{~h} \\
\text { - Noradrenalin }<1 \mathrm{mg} / \mathrm{h}\end{array}$ \\
\hline $\begin{array}{l}\text { Hirnfunktion (bei Schädel- } \\
\text { Hirn-Trauma) }\end{array}$ & $\begin{array}{l}\text { Hirndruck }<20 \mathrm{mmHg} \text { trotz Lagerung bzw. keine klinischen Hirndruckzeichen (Stammhirnreflexe, GCS, } \\
\text { Pupillenreaktion) }\end{array}$ \\
\hline Gerinnung & $\begin{array}{l}\text { - Thrombozytenzahl > 100/nl und steigend } \\
\text { - globale Gerinnungstests ohne Substitution (nahezu) normal }\end{array}$ \\
\hline
\end{tabular}

Ebenso bei der Frakturversorgung, die zu den Sekundäroperationen gehören, gibt es Unterschiede in der Festlegung der Prioritäten. Wirbelsäulen-, Becken- oder hüftgelenksnahe Frakturen sollten i.d. R. dringender definitiv versorgt werden als bspw. eine Radius- oder Unterschenkelfraktur, die zunächst ausreichend mit einem Fixateur externe ruhiggestellt sind. Generell tendiert man dazu, Frakturen, die eine Mobilisation oder Pflege des Patienten erschweren, so frühzeitig wie möglich zu versorgen. Vor allem stammnahe Frakturen, wie Wirbelsäulen- oder Beckenfrakturen, verhindern eine adäquate Pflege, Physiotherapie und Mobilisierung. Auf die Besonderheiten bei Wirbelsäulenfrakturen mit Neurologie wird weiter unten eingegangen. Auch nach adäquater Versorgung mit einem Fixateur externe kann die Versorgung eines Patienten erschwert sein. Der Patient hat Schmerzen an den Eintrittsstellen der Steinmann-Nägel, die Extremitäten lassen sich aufgrund des schweren Metalls und der fehlenden Kraft nicht gut bewegen. Die Eintrittsstellen der Steinmann-Nägel bieten Eintrittspforten für Infektionen. Die Beübung durch die Physiotherapie kann häufig erst verspätet in dem erforderlichen Maß erfolgen. Es wird immer mehr dazu übergegangen, eine frühzeitige endgültige Versorgung der Frakturen anzustreben. Dies erleichtert die adäquate Mobilisation, dadurch wiederum das Weaning von der Beatmung und minimiert das Risiko von Begleiterkrankungen [11, 12,13].

\section{Osteosynthese von Rippenfrakturen}

Vor diesem Hintergrund muss auch diskutiert werden, ob Rippenfrakturen bei Thoraxwandinstabilität (flail chest) osteosynthetisch versorgt werden sollten. Die Thoraxwandinstabilität erzeugt Schmerzen, erschwert die Sekretmobilisation und das Abhusten, die Mobilisation des Patienten und die Entwöhnung von der Beatmung und verlängert den Intensivaufenthalt. Man erhofft sich von einer frühzeitigen operativen Stabilisierung dementsprechend eine Verkürzung der Zeit am Ventilator und auf der Intensivstation, ggf. sogar Reduktion der Pneumonierate und der Sterblichkeit. In einer Metaanalyse von 2015 [15] wurden 3 prospektiv randomisierte Studien mit insgesamt 123 Patienten zusammengefasst. Es konnte eine Reduktion der Beatmungszeit und des Intensivaufenthaltes nachgewiesen werden, jedoch nicht bei der Sterblichkeit.

Um die möglichen Vorteile einer Rippenosteosynthese zu erreichen, sollte die Stabilisierung möglichst frühzeitig erfolgen. Wenn eine Thorakotomie primär erfolgt, kann im Rahmen des Eingriffs die eigentlich nicht in die Primärphase gehörende Rippenstabilisierung direkt mit umgesetzt werden. Wenn der Patient vital in den ersten Tagen nach Trauma für eine Sekundär-OP nicht operabel ist, erscheint der Sinn der Rippenstabilisierung als zusätzliche operative Maßnahme sehr fraglich [16,17].

\section{Wirbelsäulenfrakturen}

Einen weiteren Sonderfall stellen Wirbelsäulenverletzungen mit fraglicher oder bewiesener Rückenmarkläsion dar. Es besteht zunächst keine direkte vitale Bedrohung, jedoch sind die neurologischen Einschränkungen für den Patienten und seine zukünftige Lebensqualität von größter Bedeutung. Aktuelle Studien zeigen ein deutlich gebessertes neurologisches Outcome von Patienten, wenn die operative Versorgung (Reposition einer Dislokation, Dekompression des Rückenmarks, konsekutive Spondylodese) der Wirbelsäulenverletzungen mit Rückenmarkläsionen frühzeitig erfolgt. Besonders bei zervikalen Verletzungen zeigt sich ein deutlich besseres neurologisches Outcome bei Patienten, die innerhalb von $24 \mathrm{~h}$ versorgt 
wurden, gegenüber jenen, die erst nach $24 \mathrm{~h}$ versorgt worden waren. Bei sowohl kompletten als auch inkompletten Rückenmarkverletzung scheint die frühe Operation ein deutlich besseres neurologisches Outcome zu zeigen als bei einer Versorgung nach $24 \mathrm{~h}$. Dies gilt sowohl für motorische als auch für sensible Funktionen, wobei sich Letztere deutlich besser erholen [18]. In einigen Studien wurden Vergleiche durchgeführt, die eine frühe (innerhalb von $24 \mathrm{~h}$ ) vs. eine sehr frühe Versorgung (innerhalb von 5-8 h) gegenüberstellen. In den ersten Arbeiten zeigen Ergebnisse jedoch ein verbessertes neurologisches Outcome für Patienten, die innerhalb von $8 \mathrm{~h}$ versorgt wurden $[19,20]$. An dieser Stelle herrscht noch keine Einigkeit darüber, ob hier eine relevante Besserung erzielt werden kann.

Auch bei thorakalen und lumbosakralen Frakturen wird in der Klinik eine frühe Versorgung angestrebt. Wenige Studien konnten zeigen, dass Patienten, die innerhalb von $24 \mathrm{~h}$ versorgt werden, ein deutlich gebessertes neurologisches Outcome haben. Diese positiven Effekte der frühen Versorgung gelten insbesondere für inkomplette Querschnittlähmungen [21-24].

Einschränkend muss an dieser Stelle gesagt werden, dass Patienten, die in den Studien sehr früh versorgt worden waren, ansonsten als hämodynamisch stabil angesehen wurden. In den durchgeführten Studien handelte es sich zumeist um Monoverletzungen der Wirbelsäule. Die Patienten waren wenig bis gar nicht vorerkrankt. Es lässt sich jedoch festhalten, dass sich die meisten Studien darüber einig sind, dass eine frühe operative Versorgung der Wirbelsäule anzustreben ist, wenn eine Operabilität im Sinne einer stabilen Gesamtsituation des polytraumatisierten Patienten besteht [20]. Bei schwerstverletzten, kreislaufinstabilen Patienten im Schock oder mit persistierender Gerinnungsstörung oder Patienten mit einer schon initial geschädigten Lunge bzw. einschränkten Lungenfunktion muss der (neurologische) Nutzen einer dekomprimierenden und stabilisierenden Operation an der Wirbelsäule gegenüber dem Risiko des Operationstraumas und dessen Folgen individuell abgewogen werden.

Wichtig festzuhalten ist auch, dass alle Studien von der Kenntnis über den neurologischen Status des Patienten ausgehen. Es stellt sich an dieser Stelle noch die Frage, wie man vorgeht, wenn ein intubierter und sedierter Patient in den Schockraum bzw. auf Intensivstation gebracht wird. Auch hier gilt eine differenzierte Betrachtung: Ist der Patient hämodynamisch stabil? Hat er ein schweres Schädel-Hirn-Trauma? Sind eine Reduktion der Sedierung und ein Aufwachversuch tolerabel? Auf welcher Höhe ist die mögliche Rückenmarkläsion und ist eine normale Atmung mit dieser zu vereinbaren? Ist anhand der vorhandenen Diagnostik von einer motorischen und/ oder sensiblen Beeinträchtigung des Patienten auszugehen? Nach Möglichkeit sollte ein frühzeitiger, ggf. nur kurzer Aufwachversuch innerhalb der ersten Stunden angestrebt werden, um den neurologischen Status adäquat beurteilen zu können.

\section{Zusammenfassung}

Die adäquate Versorgung eines polytraumatisierten Patienten ist nach wie vor eine individualisierte und anspruchsvolle Aufgabe. Die ersten Entscheidungen sollten nach dem Leitsatz: „Treat first, what kills first“ getroffen worden. An dieser Stelle gibt es wenig Raum für freie Entscheidungen. Schwierig wird es dann, im Nachgang zu beurteilen, ab wann ein Patient für die definitive Versorgung von Frakturen operationsfähig ist. Hier wird immer mehr eine möglichst frühzeitige endgültige Versorgung angestrebt, um den Patienten schnellstmöglich mobilisieren zu können, das Risiko für Folgekomplikationen zu minimieren und generell die Intensivzeit zu verkürzen. Grundsätzlich gilt, stammnahe Frakturen werden zuerst versorgt (Wirbelsäule und Beckenring), danach die großen Röhrenknochen der Extremitäten (Oberarm/Oberschenkel) und anschließend dann die weiteren Frakturen. Die Behandlung der gleichzeitig bestehenden Weichteilverletzungen (offen > Kompartment > geschlossen) nehmen hier einen besonderen Stellenwert ein.

Eine Sonderstellung nehmen Wirbelsäulenverletzungen mit und ohne Rückmarkläsionen ein. Neuere Studien belegen, dass das neurologische Outcome eines Patienten bei frühzeitiger (unter $24 \mathrm{~h}$, nach Möglichkeit sogar unter $8 \mathrm{~h}$ ) Versorgung signifikant besser ist, sofern eine Operabilität besteht

\section{Interessenkonflikt}

Die Autorinnen/Autoren geben an, dass kein Interessenkonflikt besteht.

\section{Autorinnen/Autoren}
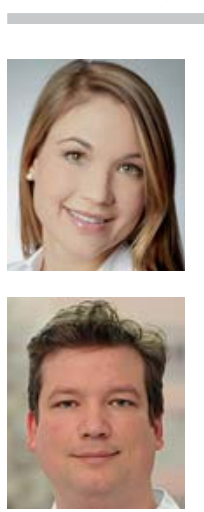

\section{Maria Alexandra Bernstorff}

Dr. med., Assistenzärztin der Chirurgischen Klinik, Chirurgische Universitätsklinik und Poliklinik, Berufsgenossenschaftliches Universitätsklinikum Bergmannsheil, Bochum

\section{Uwe Hamsen}

Dr. med., Oberarzt der Chirurgischen Intensivstation, Chirurgische Universitätsklinik und Poliklinik, Berufsgenossenschaftliches Universitätsklinikum Bergmannsheil, Bochum

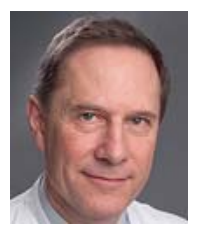

\section{Christian Waydhas}

Prof. Dr. med., Oberarzt der Chirurgischen Intensivstation, Chirurgische Universitätsklinik und Poliklinik, Berufsgenossenschaftliches Universitätsklinikum Bergmannsheil, Bochum 


\section{Dr. med. Maria Alexandra Bernstorff}

Chirurgische Universitätsklinik und Poliklinik

Berufsgenossenschaftliches Universitätsklinikum

Bergmannsheil

Bürkle de la Camp Platz 1

44789 Bochum

Tel.: 02 34/3 02-0

Fax: 02 34/302-6542

maria.bernstorff@bergmannsheil.de

\section{Literatur}

[1] Deutsche Gesellschaft für Unfallchirurgie, Hrsg. S3-Leitlinie Polytrauma/Schwerverletzten-Behandlung. Im Internet: https://www.awmf.org/uploads/tx_szleitlinien/012-019l_S3_ Polytrauma_Schwerverletzten-Behandlung_2017-08.pdf; Stand: 29.11 .2019

[2] Klüter $T$, Lippross $S$, Oestern $S$ et al. [Operative treatment strategies for multiple trauma patients: early total care versus damage control]. Chirurg 2013; 84: 759-763. doi:10.1007| s00104-013-2478-z

[3] Riska EB, von Bonsdorff H, Hakkinen S et al. Primary operative fixation of long bone fractures in patients with multiple injuries. J Trauma 1977; 17: 111-121

[4] Seibel R, LaDuca J, Hassett JM et al. Blunt multiple trauma (ISS36), femur traction, and the pulmonary failure-septic state. Ann Surg 1985; 202: 283-295

[5] Rotondo MF, Schwab CW, McGonigal MD et al. 'Damage control': an approach for improved survival in exsanguinating penetrating abdominal injury. J Trauma 1993; 35: 375-382

[6] Shapiro MB, Jenkins DH, Schwab CW et al. Damage control: collective review. J Trauma 2000; 49: 969-978

[7] Nicola R. Early total care versus damage control: current concepts in the orthopedic care of polytrauma patients. ISRN Orthop 2013; 2013: 329452. doi:10.1155/2013/329452

[8] Rostami E, Engquist H, Enblad P. Imaging of cerebral blood flow in patients with severe traumatic brain injury in the neurointensive care. Front Neurol 2014; 5: 114. doi:10.3389/ fneur.2014.00114

[9] Guo J, Yin Y, jin L et al. Acute compartment syndrome: cause, diagnosis, and new viewpoint. Medicine (Baltimore) 2019; 98 : e16260. doi:10.1097/MD.0000000000016260

[10] Waydhas C, Nast-Kolb D, Trupka A et al. Posttraumatic inflammatory response, secondary operations, and late multiple organ failure. J Trauma 1996, 40: 624-631

[11] Guerado E, Bertrand ML, Cano JR et al. Damage control orthopaedics: state of the art. World J Orthop 2019; 10: 1-13. doi:10.5312/wjo.v10.i1.1

[12] Nahm N], Vallier HA. Timing of definitive treatment of femoral shaft fractures in patients with multiple injuries: a systematic review of randomized and nonrandomized trials. J Trauma Acute Care Surg 2012; 73: 1046-1063

[13] Rixen D, Steinhausen E, Sauerland S et al.; members of the Damage Control Study Group. Randomized, controlled, twoarm, interventional, multicenter study on risk-adapted dam- age control orthopedic surgery of femur shaft fractures in multiple-trauma patients. Trials 2016; 17: 47

[14] Pape HC, van Griensven M, Rice J et al. Major secondary surgery in blunt trauma patients and perioperative cytokine liberation: determination of the clinical relevance of biochemical markers. J Trauma 2001; 50: 989-1000

[15] Cataneo AJ, Cataneo DC, de Oliveira FH et al. Surgical versus nonsurgical interventions for flail chest. Cochrane Database Syst Rev 2015; (7): CD009919. doi:10.1002/14651858. CD009919.pub2

[16] Majeed FA, Zafar U, Imtiaz T et al. Rib fixation versus conservative management of rib fractures in trauma patients. J Ayub Med Coll Abbottabad 2018; 30: 576-584

[17] Olland A, Puyraveau M, Guinard S et al. Surgical stabilization for multiple rib fractures: whom the benefit? -a prospective observational study. I Thorac Dis 2019; 11 (Suppl. 2): S130S140

[18] Fehlings MG, Vaccaro A, Wilson JR et al. Early versus delayed decompression for traumatic cervical spinal cord injury: results of the surgical timing in acute spinal cord injury study (STASCIS). PLoS One 2012; 7: e32037. doi:10.1371/journal. pone.0032037

[19] Mattiassich G, Gollwitzer M, Gaderer F et al. Functional outcomes in individuals undergoing very early $(<5 \mathrm{~h})$ and early (5-24h) surgical decompression in traumatic cervical spinal cord injury: analysis of neurological improvement from the Austrian Spinal Cord Injury Study. J Neurotrauma 2017; 34: 3362-3371. doi:10.1089/neu.2017.5132

[20] Lee DY, Park Y], Song SY et al. The importance of early surgical decompression for acute traumatic spinal cord injury. Clin Orthop Surg 2018; 10: 448-454. doi:10.4055/cios.2018.10.4.448

[21] Landi A, Marotta N, Ambrosone A et al. Correlation between timing of surgery and outcome in thoracolumbar fractures: does early surgery influence neurological recovery and functional restoration? A multivariate analysis of results in our experience. Acta Neurochir Suppl 2017; 124: 231-238. doi:10.1007/978-3-319-39546-3_35

[22] Du JP, Fan Y, Liu JJ et al. Decompression for traumatic thoracic/ thoracolumbar incomplete spinal cord injury: application of $\mathrm{AO}$ spine injury classification system to identify the timing of operation. World Neurosurg 2018; 116: e867-e873. doi:10.1016/j.wneu.2018.05.118

[23] Verheyden AP, Spiegl U], Ekkerlein $\mathrm{H}$ et al. Treatment of Fractures of the thoracolumbar spine: recommendations of the spine section of the German Society for Orthopaedics and Trauma (DGOU). Global Spine J 2018; 8 (2 Suppl.): 34S-45S

[24] Ter Wengel PV, Martin E, De Witt Hamer PC et al. Impact of early ( $<24 \mathrm{~h}$ ) surgical decompression on neurological recovery in thoracic spinal cord injury: a meta-analysis. J Neurotrauma 2019; 36: 2609-2617. doi:10.1089/neu.2018.6277

Bibliografie

DOI https://doi.org/10.1055/a-0970-9893

Online-publiziert 08.01.2020 | OP-JOURNAL 2020; 36: 30-35

(c) Georg Thieme Verlag KG Stuttgart · New York

ISSN 0178-1715 